\title{
Necessidades Educativas Especiais e funcionamento parental: indicadores de bem-estar e suporte social
}

\author{
Sara Felizardo, Esperança Ribeiro \\ Escola Superior de Educação de Viseu, CI\&DETS, Instituto Politécnico de Viseu, Portugal
}

\begin{abstract}
Resumo
O presente estudo enquadra-se numa linha de investigação que analisa a influência do suporte social no funcionamento parental em famílias de crianças com Necessidades Educativas Especiais. Foram definidos os objetivos: i) comparar os subgrupos de pais de crianças com NEE quanto ao suporte social e bem-estar; ii) compreender as relações entre as variáveis. Trata-se de uma investigação não experimental e correlacional e a amostra é constituída por 152 pais. Constatámos que existem diferenças significativas entre os subgrupos de pais (os progenitores das crianças com autismo apresentam valores mais elevados no suporte social e bem-estar) e correlações significativas e positivas entre as variáveis.
\end{abstract}

Palavras chave: inclusão, bem-estar, satisfação com a vida, suporte social, necessidades educativas especiais

\section{Introdução}

O presente estudo tem como referenciais estruturantes as perspetivas ecológica, sistémica familiar e do suporte social (Dunst, Trivette \& Jodry, 1997), sendo o nosso propósito refletir sobre o papel do suporte social nas dinâmicas de desenvolvimento e no funcionamento parental em famílias de crianças com Necessidades Educativas Especiais (NEE), particularmente, o seu efeito no bem-estar dos pais/ cuidadores, no âmbito do processo de adaptação parental à deficiência e incapacidade. As linhas atuais de investigação, assim como as experiências relatadas pelos pais e técnicos dos serviços de apoio têm colocado, consistentemente, em causa a validade universal e normativa da perspetiva deficitária, centrada nos aspetos patológicos das famílias de crianças e jovens com deficiência e incapacidade (Felizardo, 2013). A literatura científica no domínio sugere que as famílias podem adaptar-se continuamente às mudanças e às circunstâncias, com períodos de (re)ajustamento ao longo das diversas etapas do ciclo da vida familiar. Assim, numa primeira fase, os pais podem enfrentar o choque pela situação inesperada; a crise que envolve um primeiro diagnóstico de que algo está mal com o filho é, provavelmente, para os pais, a maior dificuldade e uma experiência de angústia. Na maior parte dos casos, as suas reações iniciais são negativas e similares às que estão relacionadas com processos de luto (Felizardo, 2009, 2013; Marvin \& Pianta, 1996). No entanto, estes pais podem desenvolver atitudes e comportamentos adaptativos, promotores de alternativas de qualidade e, por conseguinte, geradores de soluções eficazes na organização dos acontecimentos da vida diária e nos processos de transição (Fine, 1991). Estas famílias são proativas e procuram resolver os problemas de forma eficaz, com os recursos que dispõem; neste sentido, assumem as diversas tarefas e exigências de educação e cuidado e, principalmente, aceitam os filhos na sua especificidade, empenhando-se no sentido de conseguir os melhores serviços e técnicos. Deste modo, procuram ultrapassar os eventuais problemas que possam surgir nos subsistemas conjugal e parental, no quadro de um ajustamento dinâmico às crises e mudanças familiares. Estas famílias referem ter uma adaptação positiva, sendo, muitas vezes, elas próprias, fontes de suporte a outras famílias com problemáticas similares (Summers, Behr \& Turnbull, 1989).

\section{Suporte social e bem-estar parental}

A literatura científica sobre o bem-estar subjetivo salienta a vastidão desta linha de investigação. É considerado um fenómeno complexo e multideterminado, composto por várias componentes, de natureza cognitiva e afetiva, abrangendo diversas dimensões e parâmetros de análise. Diener (2009) apresenta uma definição de bem-estar subjetivo como sendo uma resposta avaliativa das pessoas relativamente à sua própria vida, quer em termos de satisfação (elemento cognitivo da avaliação), quer em termos de afetividade (elemento emocional estável). Neste contexto, o valor potencial do suporte social atraiu a atenção dos teóricos e técnicos no âmbito dos temas relacionados com a saúde física e mental, onde $\mathrm{o}$ construto adquiriu um papel central como um fator explicativo no contexto da perspetiva da ecologia humana do comportamento e desenvolvimento da criança e da família (Felizardo, 2013). O suporte social é considerado um processo complexo, dinâmico e transacional, que envolve a troca de recursos entre um indivíduo e os membros da sua rede social. Pode ser definido como uma transação interpessoal de ajuda e assistência emocional, psicológica, informativa, instrumental ou material, proporcionada pelos membros da rede social que influenciam de uma forma positiva o comportamento (Dunst, Trivette \& Deal, 1994). O trabalho de Dunst e colaboradores (Dunst, Trivette e Jodry, 1997) revelou que o sistema de suporte social dos pais pode não só operar efetivamente para influenciar um funcionamento parental mais positivo, mas pode também resultar num desenvolvimento mais favorável e adaptativo da criança. Apesar de tudo, parece claro que a principal função dos sistemas de suporte é proporcionar uma sustentação parental mais sólida, a partir da qual os pais podem, por seu turno, facilitar um desenvolvimento da criança mais positivo. Dunst, Trivette e Jodry (1997), 
apoiando-se nos diversos estudos neste domínio, sustentam que o suporte social e os recursos influenciam a saúde, o bem-estar e têm implicações no funcionamento familiar.

\section{Método}

Foram definidos os seguintes objetivos: i) proceder a análises diferenciais entre os subgrupos de pais ou cuidadores de crianças com NEE (crianças com incapacidade intelectual; crianças com problemas motores e crianças com perturbações do espectro do autismo) nas variáveis suporte social e satisfação com a vida; ii) compreender as relações entre as variáveis de suporte social e satisfação com a vida. Trata-se de uma investigação com um plano não experimental e correlacional.

\section{Participantes}

A amostra é de conveniência, sendo constituída por 152 pais, com três subgrupos: 82 pais de crianças com incapacidade intelectual, 37 pais de crianças com problemas motores e 33 pais de crianças com autismo.

\section{Instrumentos}

Os instrumentos de recolha foram a Escala de Satisfação com a Vida - SV (Neto, Barros \& Barros, 1990; Simões, 1992), que avalia a dimensão cognitiva do bem-estar; o Questionário de Suporte Social - SSQ6 (Pinheiro \& Ferreira, 2001), que contempla duas subescalas: SSQ6N (disponibilidade/ extensão do apoio) e SSQ6S (satisfação com o apoio); um Questionário Parental (dados sociodemográficos e familiares).

\section{Procedimentos}

Os dados foram recolhidos em agrupamentos de escolas e em instituições de apoio à deficiência localizadas numa cidade da região centro de Portugal. Para o efeito, foram feitos os pedidos de autorização e apoio, a saber: Ministério da Educação; Diretores dos Agrupamentos de Escolas e das Instituições; professores de Educação Especial e pais das crianças com NEE.

\section{Resultados}

Tendo em atenção os objetivos propostos, constatamos que no suporte social, as subescalas SSQ6N e SSQ6S apresentam correlações significativas e positivas com o bem-estar/ satisfação com a vida $(\mathrm{r}=.539 ; \mathrm{r}=.695 ; p \leq$ .001). Estes resultados são consistentes com os estudos de validade do suporte social de Saranson, Levine, Basham e Saranson (1983), onde foram encontradas correlações significativas negativas com medidas de ansiedade e depressão, bem como, correlações significativas positivas com variáveis do bem-estar psicológico, em particular, nas relações positivas com os outros, no desenvolvimento pessoal e na satisfação (Pavot \& Diener, 2009). Tal como constatamos na Tabela 1, os resultados dos pais das crianças com PEA no suporte social, SSQ6N (M=19.51;DP=8.34) e SSQ6S $(\mathrm{M}=29.12$; $\mathrm{DP}=6.41)$ e $\mathrm{SV}(\mathrm{M}=18.63 ; \mathrm{DP}=3.16)$ foram os mais elevados.
Tabela 1. Resultados dos pais no Suporte Social e Satisfação com a vida (Médias e Desvios-Padrão)

\begin{tabular}{lcccccc}
\hline Medidas & \multicolumn{2}{c}{ Pais - PrI } & \multicolumn{2}{c}{ Pais - PrM } & \multicolumn{2}{c}{ Pais - PEA } \\
& M & DP & M & DP & M & DP \\
\hline $\begin{array}{l}\text { SSQ6 N } \\
\begin{array}{l}\text { Número } \\
\text { SSQ6 }\end{array}\end{array}$ & 13.37 & 7.92 & 13.27 & 6.35 & 19.51 & 8.74 \\
Satisfação & 25.41 & 8.50 & 26.62 & 5.86 & 29.12 & 6.41 \\
\hline \multicolumn{3}{c}{ Teste Scheffé - SSQ6N - PEA >PrI; PEA>PrM } \\
\hline $\begin{array}{l}\text { Satisfação } \\
\text { com a vida }\end{array}$ & 15.74 & 4.59 & 16.48 & 4.37 & 18.63 & 3.16 \\
\hline
\end{tabular}

A pesquisa das relações entre o tipo de problemática e as medidas de suporte social e satisfação com a vida centrou-se na análise da variância (one way anova). Os resultados revelam que existem diferenças significativas entre os subgrupos de pais de crianças $(p \leq .001)$ com Problemas Intelectuais (PrI), Motores (PrM) e Perturbações do Espectro do Autismo (PEA). A análise mais detalhada dos resultados, com comparações post-hoc, do Teste Scheffé, revela que, no suporte social relativo à extensão da rede (SSQ6N), as diferenças ocorrem entre as PEA, face aos $\operatorname{PrI}(p \leq .001)$ e $\operatorname{PrM}(p \leq$ $.001)$, ou seja, PEA $>$ PrC; PEA $>$ PrM

\section{Discussão}

Os resultados relativos ao efeito do tipo de NEE revelam que os pais das crianças com autismo (PEA) apresentam valores significativamente mais elevados no suporte social (disponibilidade/ extensão do suporte e satisfação com o suporte) e na satisfação com a vida. Estes dados são, de algum modo, surpreendentes, pois a literatura científica acentua que os pais das crianças com autismo parecem ter fontes específicas de stress associadas à variedade de sintomas e problemas comportamentais (Benson \& Karlof, 2009). A estes resultados não será alheio o facto de estes pais estarem ligados a uma Associação Portuguesa para as Perturbações do Desenvolvimento e Autismo da região onde foi efetuado o estudo, que proporciona um bom suporte e funciona de uma forma muito próxima com os pais ou cuidadores. De forma convergente, os estudos de Heiman (2000) sobre famílias resilientes de crianças com problemas de desenvolvimento salientaram a importância dos recursos sociais e os efeitos dos vários tipos de suporte nas respostas às necessidades das famílias. $\mathrm{Na}$ atualidade, a maioria dos programas de intervenção oferecem uma variedade de níveis de suporte direcionados primordialmente para os pais e, não tanto, diretamente às crianças. Este modelo de atendimento centrado nos pais ou cuidadores reconhece que estes constituem o principal contexto de desenvolvimento da criança e, neste sentido, o funcionamento parental poderá ser fortemente preditivo do desenvolvimento subsequente da criança. Os pais são a primeira e principal fonte de suporte emocional para a criança e, por conseguinte, os mais competentes estão também mais capacitados para satisfazer as necessidades da criança (Gupta \& Singhal, 
2004). Os maiores desafios para as linhas de investigação futuras estão relacionados com o estudo dos mecanismos envolvidos na forma como as variáveis do suporte social se influenciam reciprocamente de modo a maximizar o impacto do suporte social no bem-estar parental, familiar e no desenvolvimento da criança.

\section{Referencias}

Benson, P.R. \& Karlof, K.L. (2009). Anger, stress proliferation, and depressed mood among parents of children with ASD: A longitudinal replication. Journal. Autism Dev. Disord., 39, 350-362.

Diener, E. (2009). Assessing subjetive well-being: Progress and opportunities. In E. Diener (Ed.), Assessing well-being.The collected works of Ed Diener. Social Indicators Research Series (pp. 25-65). London New York: Springer.

Dunst, C.J.; Trivette, C.M. \& Deal, A.G. (1994). Supporting and strengthening families: Methods, strategies and practices. Cambridge, MA: Brookline Books.

Dunst, C.J.; Trivette, C.M. \& Jodry, W. (1997). Influences of social support on children with disabilities and their families. In M.J. Guralnick (Ed.), The effectiveness of early intervention (pp. 499-522). Baltimore, Maryland: Paul H. Brooks.

Felizardo, S.M.A.S. (2009). O impacto do suporte social em famílias de crianças com deficiência. International Journal of Development and Educational Psychology, XXI, 1 (3), 267-274.

Felizardo, S.M.A.S. (2013). Deficiência, família(s) e suporte social: contextos e trajetórias de desenvolvimento para a inclusão. Tese do Doutoramento. Universidade de Coimbra (Faculdade de Psicologia e Ciências da Educação). http://hdl.handle.net/10316/21833

Fine, M. J. (1991). The handicapped child and the family: Implications for professionals. In M. J. Fine (Ed.), Colaboration with parents of exceptional children (pp. 3-24). Brandon, Vermont: CPPC (Clinical Psychology Publishing Company).
Gupta, A. \& Singhal, N. (2004). Positive perceptions in parents of children with disabilities. Asia Pacific Disability Rehabilitation Journal, 15 (1), 22-35.

Heiman, T. (2002). Parents of children with disabilities: Resilience, coping and future expectations. Journal of Developmental and Physical Disabilities, 14 (2), 159-171.

Marvin, R.S. \& Pianta, R.C. (1996). Mothers`reactions to their child's diagnosis: Relations with security and attachment. Journal Clinic Child Psychology, 25, 436-445.

Pavot, W. \& Diener, E. (2009). Review of the satisfaction with life scale. In E. Diener (Edit.), Assessing well-being. The collected works of Ed Diener. Social Indicators Research Series (pp. 101-117). London New York: Springer.

Pinheiro, M.R.M. \& Ferreira, J.A.G. (2002). O questionário de suporte social: Adaptação e validação do SSQ6. Psychologica, 30, 315-333.

Simões, A.; Ferreira, J.A.; Lima, M.P.; Pinheiro, M.R.M.M.; Vieira, C.M.C.; Matos, A.P.M. \& Oliveira. (2000). O bem-estar subjetivo: Estado atual dos conhecimentos. Psicologia, Educação e Cultura, Vol. IV, 2, 243-279.

Summers, J.A.; Behr, S.K. \& Turnbull, A.P. (1989). Positive adaptation and coping strengths of families who have children with disabilities. In G.H.S. Singer \& L.K. Irvin (Eds.), Support for caregiving families: Enabling positive adaptation to disability (pp 27-40). Baltimore: Paul H. Brookes. Publishing Co. 